\title{
Generating Fractalstructure and Route Matrix in Zodiac Signs
}

\author{
R. Kamali, G. Jayalalitha
}

\begin{abstract}
This paper deals with finding out the possibilities of generating the Fractal Structure in the Zodiac Signs. Fractals are a continuous complex pattern which is Self-Similar across different scales; these Signs in the Natal Chart are also create a Self-Similar structure which is continuous too. The positions of the planets in the zodiac signs have different combinations and these combinations are captured in Graph. The connections of the planets in the Natal Chart are generated in the form of Route Matrix. This matrix is also useful for the Astrologers in predicting one's future.
\end{abstract}

Keywords: Complex pattern, Dynamical Systems, Local Structure, Fractals, Zodiac, Route Matrix.

AMS Classification: 30D05, 30D40, $70 F 15$

\section{INTRODUCTION}

An ancient study of planets position and stars location in the Universe is known as Astrology which is based on the Natal Chart i.e. Horoscope. This chart is nothing but the diagrammatic representation of the various positions of the planets on the time and place of birth of each and every individual. The position of the planets in relation to the place of birth of an individual states their expression in the spheres of life which is described by the Astrological houses $[1,2]$. The planetary force takes different form, depending on their Zodiacal position and on the way they relate to one another. Hence there is an irregular formation of planets in one's horoscope which is repeated continuously throughout their life time.

Fractal is a continuous complex pattern that is self-similar across different scales. They are created by repeating a simple process over and over in an ongoing feedback loop. The horoscope forms as a fractal because of

an irregular pattern in the formation of planets which is continuous too. Driven by recursion, fractals are images of dynamical systems as the Universe is dynamical in nature, the pictures of Chaos which deals with nonlinear things that are effectively impossible to predict. Also, this universe is a dynamical system in which the motion of the planets in the Zodiac sign describes the time dependence of a point in a geometrical space [3, 4]. These phenomena are often described by Fractal mathematics, which captures the infinite complexity of nature.

Revised Manuscript Received on December 5, 2019.

Revised Version Manuscript

R. Kamali, Research Scholar, Department of Mathematics, VELS Institute of Science, Technology and Advanced Studies, Chennai, Tamil Nadu, India. (e-mail: kamali_1883@yahoo.co.in)

G. Jayalalitha, Professor, Department of Mathematics, VELS Institute of Science, Technology and Advanced Studies, Chennai, Tamil Nadu, India. (e-mail: g.jayalalithamaths.sbs@velsuniv.ac.in
The motion of the planets play a vital role in Astrology $[5,6]$, during this motion of planets there is a possibility for the occurrence of the tangents by these planets to the Earth. Each and every person's horoscope has a structure of planets which is not unique [7, 8]. By the definition of tangents, there forms a Local Structure for the planets [9, 10], since the Structures are not uniqueness with the positive measure in the Zodiac. The placement of planets in the 12 houses reveals the energy, as well the strengths and weaknesses of the planets.

Also in one's horoscope there forms an extensive relationship among these planets in the 12 houses, due to this connection of the planets, there exists a path between the planets which leads to a route $[11,12]$, as route indicates the connection between any two, this can be expressed in Matrix form, a mathematical tool which is a simpler way of representing any information through numerical value to solve the needs. This connection of planets in the Zodiac leads to the formation of the Route Matrix.

\section{ZODIAC AS LOCAL STRUCTURE}

In general the Universe is dynamical in structure; there is a constant change among the motion of the planets and stars in a horoscope. On the whole, Zodiac is a nothing but the twelve-part division of the sky named as Signs with size $30^{\circ}$ each as in Fig.-1 with certain Astrological properties. These 12 Signs of Zodiac came to existence by projecting consciousness, represented by the planets into time and space.It is further developed into two systems namely, Tropical Zodiac and Sidereal Zodiac.

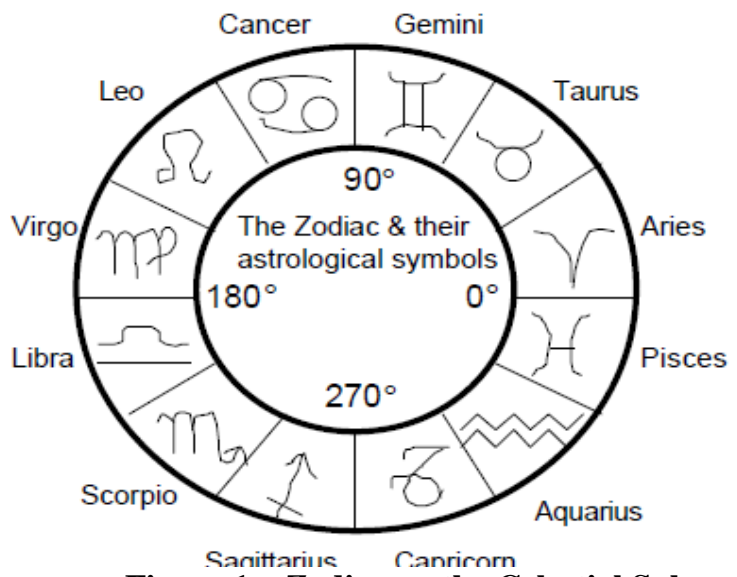

Figure 1 Zodiac on the Celestial Sphere

In the Zodiac there exists a very slow process of motion of planets known as Precession. The Precession shifts Vernal equinox between stars and makes the Earth's axis of rotation to vibrate. When these constellations coincide exactly with Zodiac, the axis of rotation sweeps out like a 
cone as shown in Fig.-2. Due to this the Celestial equator is perpendicular to the axis. This shows that these Celestial equator moves along with the axis of rotation and the ecliptic is unaffected by this.

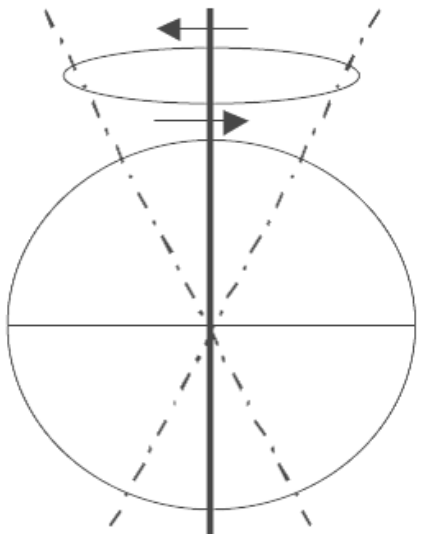

Figure 2 Precession

Based on the movement of the planets in zodiac, the natal chart drawn during at a particular time is suitable for all the people who are born approximately in that time with a lack of distinctive character among the other people. Therefore, in order to create Zodiac more individual for all, a Local element with twelve parts is introduced. This Local element is familiarly known as House division with twelve houses namely I, II... XIIas shown in Fig.-3.At the same time as the Sun makes its revolution it resides in each of the twelve houses. A person born in certain time will attain the sign of a house that contains the Sun in that time. This sign is called as the Sun sign or the Zodiac sign of that person. Then that person is believed to have the characteristics associated with that sign.

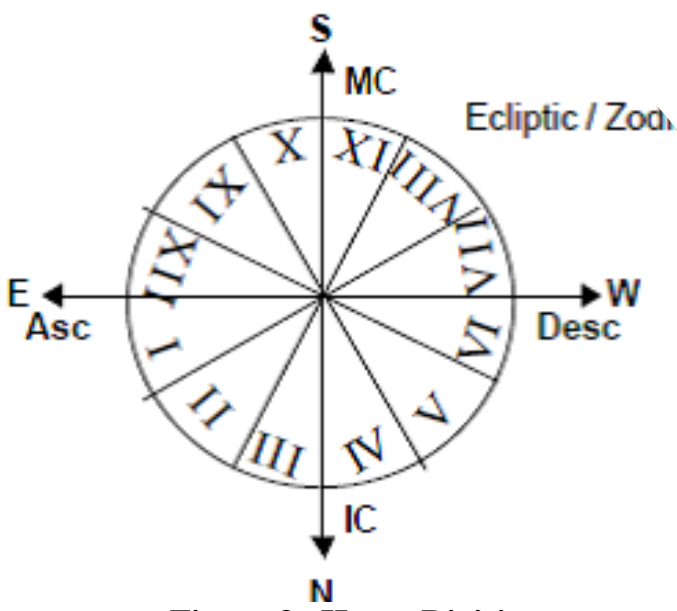

Figure 3 House Divisions

Usually, the movement of the planetsaround the sun takes eastwards but sometimes shifts westwards, which is known as Retrograde or Backwards motion. The path described by one Celestial body in its revolution about another around the Sun, is much bigger when compared to the path described by the earth, which is tangential. The entire period for retrograde motion will differ for each and every planet. Hence there exists an irregular pattern of motion among the planets which includes the existence of tangents and leads to the structure of fractals sets for this irregular motion, let it be an s-sets.

For this s-sets the generalization of tangents should reflect the local directional distribution of sets of positive measure of planet's motion which are complexity and for a subset $F$ of these s-sets there exists a tangent at a distance $x$ in direction $\theta$, where $\theta$ is a unit vector, if the density of the sset is denoted as $\bar{D}^{s}$ is given as

$$
\bar{D}^{s}(F, x)>0
$$

and for every angle $\varphi>0$, the limit of the s-set is defined as

$$
\lim _{r \rightarrow 0} r^{-s} \mathcal{H}^{s}(F \cap[B(x, r) \backslash S(x, \theta, \varphi)])=0
$$

where $\mathcal{H}^{s}$ is the Hausdorff Space of s-set, $B(x, r)$ is a closed disc of radius $r$ and centrex, $S(x, \theta, \varphi)$ is a double sector with vertex $x$, containing $y$ such that the line segment $[x, y]$ makes an angle at most $\varphi$ with $\theta$ or $-\theta$ as in Fig. -4 . This double sector resembles the precession occurred in zodiac sign with certain angles.

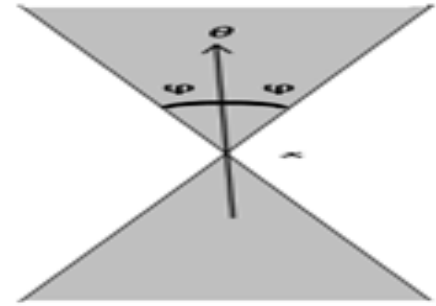

Figure 4 Double sector $S(x, \theta, \varphi)$

Generally fractals are defined as a set with Hausdorff dimension strictly greater than its topological dimension. Thus for a tangent in the direction $\theta$, eqn.-(1) requires a significant part of $F$ lying near $x$. By eqn.-(2) a negligible amount close to $x$ lies outside the double sector $S(x, \theta, \varphi)$ as in Fig.-5. The existence of this tangential in the zodiac sign is confirmed by the following Proposition.

\section{Proposition 2.1}

Rectifiable curve $C$ has tangents at almost all of its points.

\section{PROOF}

Let us consider a horoscope as a rectifiable curve $C$ with $\mathcal{L}(\mathrm{C})<\infty$. This is because the universe has distinct end points say $p$ and $q$ for which $\mathcal{L}(\mathrm{C}) \geq|p-q|>0$.

Also the Hausdorff dimension for 1 -set is given as $0<\mathcal{H}^{1}(\mathrm{C})<\infty$, this shows that $C$ is a 1 -set and the density for this 1 -set, $D^{1}(C, x)$ exists and equals 1 and the upper density $\bar{D}^{1}(C, x)>0$ for almost all $x \in C$.

These densities relate the planet combinations in different houses in a horoscope; some houses have one planet and some houses may have one or more planets.

It is possible to re-parametrize the curve $C$ by arc length, such that $\psi:[0, \mathcal{L}(\mathrm{C})] \rightarrow \mathbb{R}^{2}$ gives $\psi(\mathrm{t})$ as the point distance $t$ along $C$ from the end point $\psi(0)$.

Also $\mathcal{L}(\mathrm{C})<\infty$ simply means that $\psi$ has bounded variation; in other words,

$\sup \sum_{i=1}^{m}\left|\psi\left(\mathrm{t}_{\mathrm{i}}\right)-\psi\left(\mathrm{t}_{\mathrm{i}-1}\right)\right|<\infty(3)$

where the supremum is over dissections $0=t_{0}<t_{1}<\cdots<$ $t_{m}=\mathcal{L}(\mathrm{C})$.

This resembles the maximum distance among the planets in a horoscope. 


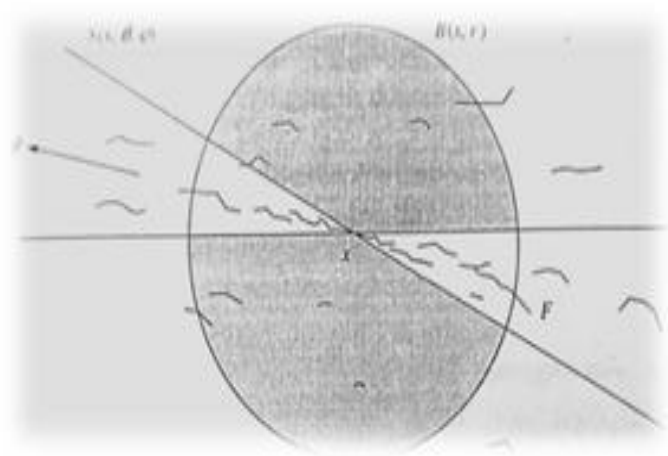

Figure 5 Tangent direction of $F$

Since the function of bounded variations are differentiable everywhere, there exists a vector $\psi^{\prime}(t)$ for almost all $t$.

Due to the parametrization in arc-length

$$
\left|\psi^{\prime}(t)\right|=1 \text { for all } t \text {. }
$$

Hence for all points $\psi(\mathrm{t})$ on $C$, there exists a unit vector $\theta$ such that

$$
\lim _{u \rightarrow t}\left[\frac{\psi(\mathrm{u})-\psi(\mathrm{t})}{\mathrm{u}-\mathrm{t}}\right]=\theta
$$

Thus, for given $\varphi>0$, there is a number $\varepsilon>0$ such that

$$
\psi(\mathrm{u}) \in S(\psi(\mathrm{t}), \theta, \varphi)
$$

whenever, $|u-t|<\varepsilon$.

As $C$ has no double points, it may find $r$ such that $\psi(\mathrm{u}) \notin B(\psi(t), r)$

if $|u-t| \geq \varepsilon$, so $C \cap[B(\psi(t), r) \backslash S(\psi(t), \theta, \varphi)]$ is empty.

Therefore from eqn.-(1) and eqn.-(2), it is clear that the curve $C$ has tangent at $\psi(\mathrm{t})$, for almost all points on $C$, as in Fig.-5.

Hence the Rectifiable curve $C$ i.e., the horoscope has a tangent at almost all of its points.

From the above proposition it is clear that the Precession in the planets forms a tangential arc which is because of the earth's revolution.

Usually the densities of irregular sets cannot be unsteady. Moreover, these densities are one of the main characteristic in the Zodiac signs to form a Local Structure. Based on this and the rotation of the planets in the Zodiac, an irregular structure is formed as in Fig.-6. Sometimes a regular structure of planets is also formed as in Fig.-7. In each figure, the connection of the planets in the Zodiac signs gives a Complex pattern. The Structure may be regular or irregular but there exists a Self- Similarity which leads to Fractal Structure.

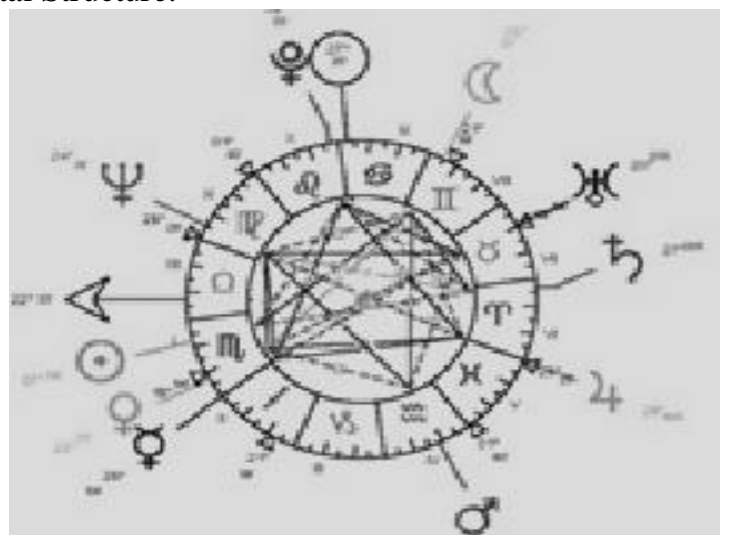

Figure 6 Irregular Structure

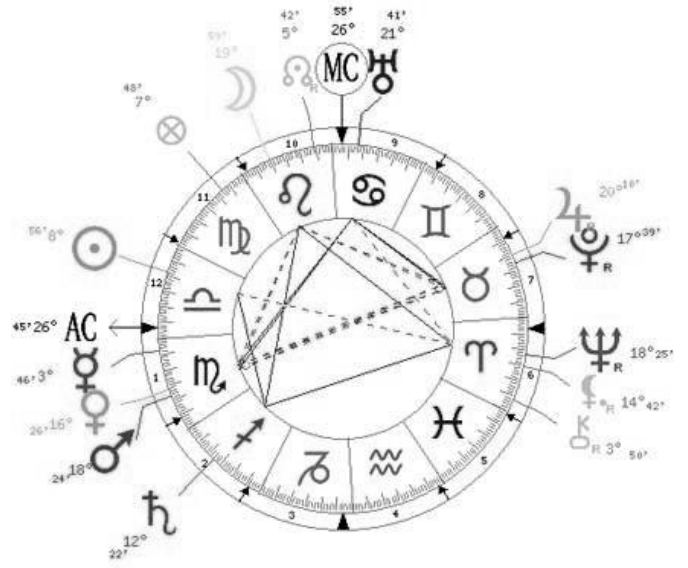

Figure 7 Regular Structure

A Self-Similarity is the subset of the object which is similar as the whole. These subsets need not look exactly the same as each other at all scales, but the same type of Structure appears at all scales. Hence the Complex pattern seen in Zodiac Signs of each and every person's horoscopes is different and shows the formation of Statistical SelfSimilarity in each horoscope which creates a Fractals Structure.

Hence the Zodiac Signs form a Local Structure of fractals with the tangential aspects for locality of planets around the Sun in the Universe.

\section{ROUTE MATRIX IN ZODIAC}

The connection of the planets in the Zodiac signs as in Fig. -6 and Fig.-7 leads to the formation of Graph which is also very useful in representing the information about the planets combinations. The significance of the planets in a birth chart is determined by the houses they occupy as each house represents an aspect of life. The distance between these planets is important since each has its own celestial function. In particular, many problems can be modeled with paths formed by traveling along the edges of a certain graph.The path in the graph induces that there forms a route among the connection of the planets.

However when there are many routes, they can become so visually complicated, that it is very difficult to see patterns. The routes thus formed is a directed route, as the directed route is a set of points $P_{l}, P_{2} \ldots P_{n}$ called vertices together with a finite set of directed edges each of which joins on ordered pair of distinct vertices. Also each edge of a directed route is called a stage of length 1 . A path from the vertex $P_{i}$ to the vertex $P_{i}$ is a sequence of directed edges from $P_{i}$ to $P_{j}$.

It is also possible to represent information about the connection of planets in the form of matrices because the directed route is represented by its route matrix. Representing the connection in this way allows the application of a mathematical tool, Matrix to summarize and find patterns. For a directed route with ' $\mathrm{n}$ ' vertices the $n \mathrm{X} n$ matrix $A$ is formed with the $(i, j) t h$ element is 1 , if there is a directed edge from $P_{i}$ to $P_{\mathrm{i}}$ and $O$ otherwise is called the route matrix. Thus, the path matrix between any two planets if there is a direct connection, is the matrix $P=\left\{P_{i j}\right\}$ such that 


$$
P=\left\{\begin{array}{c}
1 \text { if there is a path from } P_{i} \text { to } P_{j}(7) \\
0 \text { otherwise }
\end{array}\right.
$$

Thus the connection among the planets in one's horoscope induces about the analyzation of Yoga's such as Rajayoga, Dhanayoga, etc. attained in their life.

For example, consider the following 4 horoscopes of 4 different persons who born in same date and time, but on different year in which the positions of the planets are discussed more clearly and were established in a route matrix as below:

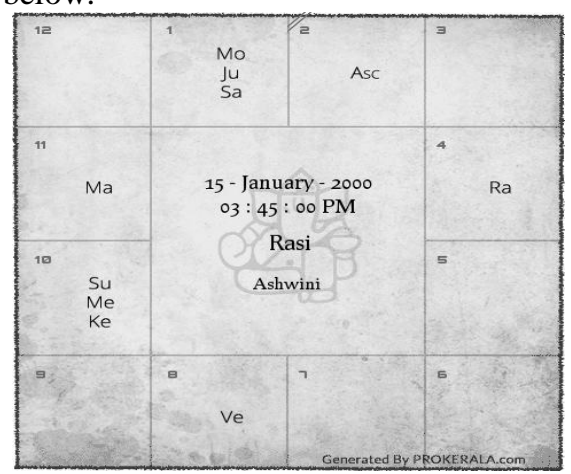

Horoscope $i$

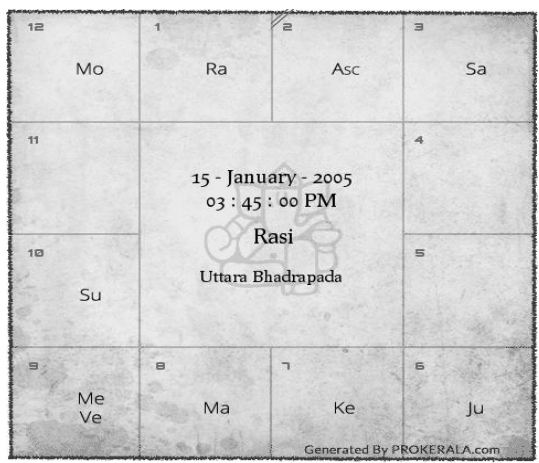

Horoscope $i i$

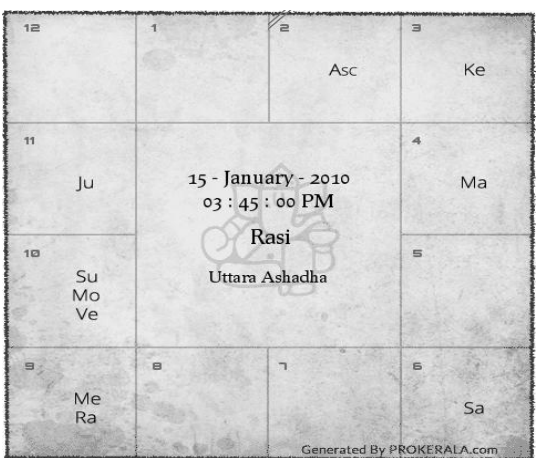

Horoscope $i i$

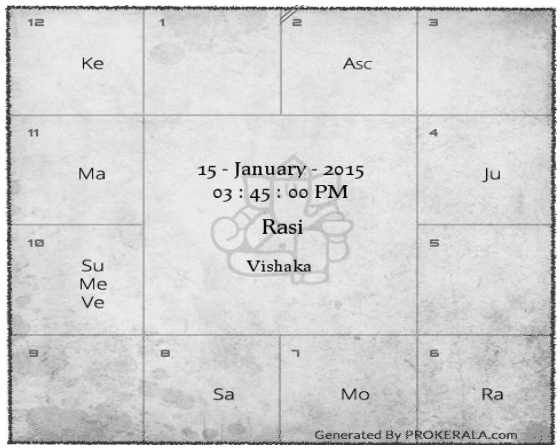

Horoscope $i v$

Figure 8 Horoscopes of 4 different persons
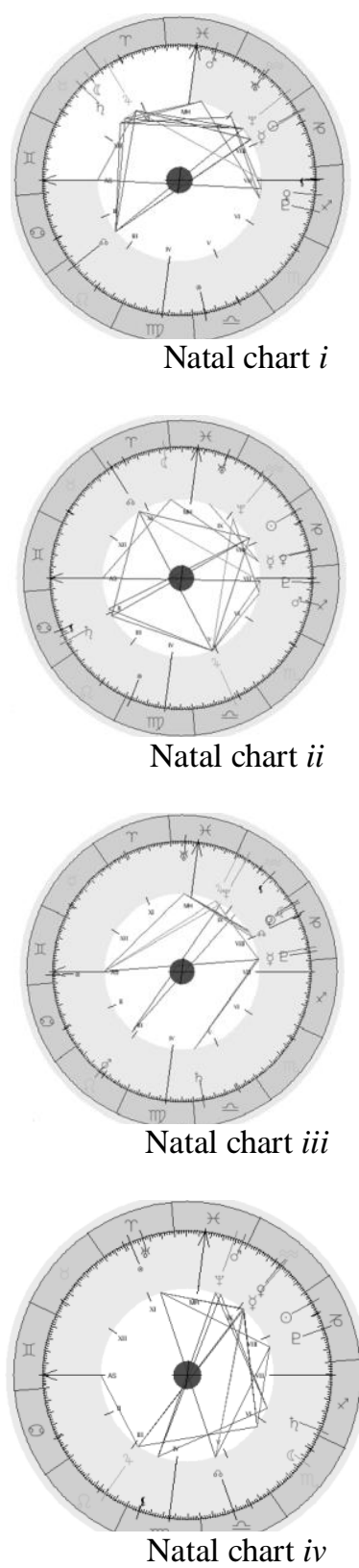

Figure 9 Natal charts of 4 different persons

For the above mentioned Horoscopes of different persons the corresponding Natal chart are thus formed due to the connection of the planets in their horoscopes as in Fig. -8 .

$P_{1}$
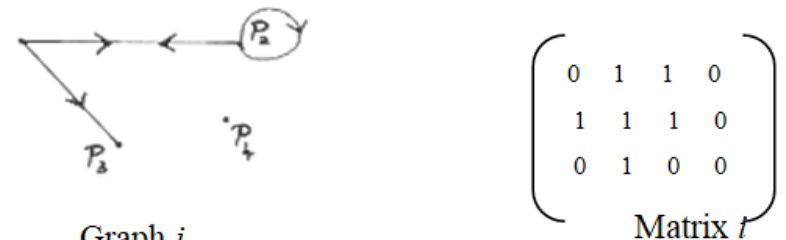

Graph $i$

Matrix $t$

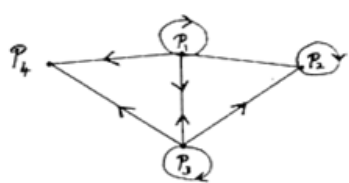

Graph ii

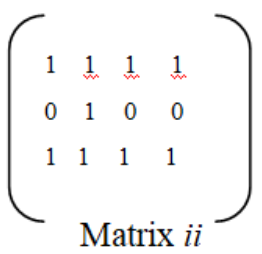

Published By:

Blue Eyes Intelligence Engineering 


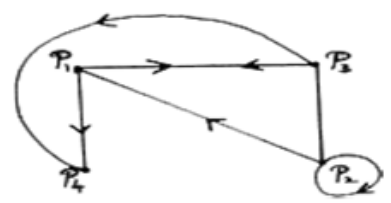

Graph iii

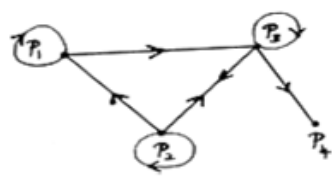

Graph iv

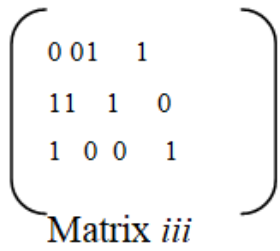

$\left(\begin{array}{ccccc}1 & 0 & 1 & 0 & \\ 1 & 1 & 1 & 0 \\ 0 & 1 & 1 & 1\end{array}\right)$

Matrix iv
Figure 10 Route Graphs and Route Matricesof 4 different persons Horoscopes

Based on the connections of the planets in the natal charts as in Fig.-9, the paths are created among the planets which lead to the formation of graphs as in Fig.-10. The graphs thus formed for these different charts are irregular due to the position of the planets in the horoscopes and these positions of the planets will differ in each person's horoscopes. The graph for each and every horoscope is highly individual and very complex since each horoscope have different planets in different signs.

The paths in the graphs $i, i i$, iii and $i v$ are connected to one another from which the formation of matrices are found based on the eqn.-(7). The corresponding matrices $i$, ii, iii and $i v$ as in Fig.-10 are the matrices of the graphs which are unique in structure since the position of the planets are different for different persons. These connected paths show the creation of routes in the graphs.

Each of these elements in the matrices can individualize places and charts forming places for each of the planets with particular signs. Hence each and every route matrix for different horoscopes are different i.e., an irregular pattern of matrices is formed this leads to the formation of fractals.

Thus the Route matrix is an effective way which interoperates each and every person's individual realities. This also gives the ability to predict on what happens throughout one's day to day life as it uses trend to predict one's life's potential.

\section{CONCLUSION}

The basic pattern of the universe repeats them self everywhere which leads to a fractal-like structure and forms a Local structure of fractals too. Consequently, the pattern that planets create reflects the ups and downs in human life. This planetary transit can be calculated by using Route matrix and compared with the birth chart in order to see how the positions, angles and aspects are influencing day to day activities in an individual's life and to make certain predictions.

\section{REFERENCES}

1. Ove H. Sehested, 1973, The Basics Of Astrology, Volume 1, Uranus

2. Publishing Co. Kevin HengSer Guan,(2000) The Mathematics Of Astrology does
3. House Division MakeSense?, National University of Singapore,

4. Undergraduate Research OpportunitiesProgramme in Science.

5. David A. Brannan, Matthew F. Esplen and Jeremy J. Gray, (1999)

6. Geometry, Cambridge University Press.

7. Falconer K., 2014, Fractal Geometry, Mathematical Foundations and Applications, ThirdEdition.

8. Ronny Martens and Tim Trachet, 1998, Making Sense of Astrology,

9. Prometheus Books.

10. www.dyna.iperwtb.com/astrology/primer_7_1.htm

11. Mattila P. Hausdorff $m$ regular and rectifiable sets in $n$-space, Trans.

12. Am. Math. Soc., 205, 263-274, (1975a).

13. Federer H.,(1996) Geometric Measure Theory, Springer, New York.

14. Besicovitch A. S. On the fundamental geometric properties of

15. linearly measurable plane sets of points II, Math. Ann., 115, 296-

16. $329,(1938)$.

17. Mastrand J.M. Some Fundamental Geometrical properties of plane

18. sets of Fractionaldimensions, Proc. Lond.Math.Soc. (3), 4, 257

19. 302,(1954a).

20. Gross J. \& Yellen, 2006, J. Graph Theory and Its Applications,

21. CRC Press.

22. H. Whitney, Congruent graphs and the connectivity of graphs, 23. Amer. J. Math. 54, 150-168, (1932). 